\title{
Effects of the self- and cross-diffusion on positive steady states for a generalized predator-prey system *
}

\author{
Yunfeng Jia ${ }^{a, \dagger}$ Pan Xue ${ }^{b}$ \\ ${ }^{a}$ School of Mathematics and Information Science, \\ Shaanxi Normal University, Xi'an, Shaanxi 710062, China \\ ${ }^{b}$ College of Computer Engineering, \\ Shaanxi Electronic Technical College, Xi'an, Shaanxi 710125, China
}

\begin{abstract}
This paper deals with a generalized predator-prey system with cross-diffusion and homogeneous Neumann boundary condition, where the cross-diffusion is included in such a way that the prey runs away from the predator. We first give a priori estimate for positive steady states to the system. Then we obtain the non-existence result of nonconstant positive steady states. Finally, we investigate the stability of constant equilibrium point and the existence of non-constant positive steady states. It is shown that the system admits a non-constant positive steady state provided that either of the self-diffusions is large or the cross-diffusion is small.
\end{abstract}

Keywords Predator-prey system; Self- and cross-diffusion; Positive steady states; Stability; Leray-Schauder degree

2000 Mathematics subject classifications 35K57; 35K60; 92B05

\section{Introduction}

In this paper, we consider the following generalized predator-prey system with constant self- and cross-diffusion rates

$$
\begin{cases}u_{t}-d_{1} \Delta\left(\left(1+d_{3} v\right) u\right)=u g(u)-p(u) v, & (x, t) \in \Omega \times(0, \infty), \\ v_{t}-d_{2} \Delta v=v(-d+c p(u)), & (x, t) \in \Omega \times(0, \infty), \\ u(x, 0)=u_{0}(x) \geq 0, v(x, 0)=v_{0}(x) \geq 0, & x \in \Omega, \\ \partial_{\nu} u=\partial_{\nu} v=0, & (x, t) \in \partial \Omega \times(0, \infty),\end{cases}
$$

where $\Omega \subset R^{N}$ is a bounded domain with smooth boundary $\partial \Omega ; \nu$ is the outward unit normal vector on $\partial \Omega$ and $\partial_{\nu}=\partial / \partial \nu$. $u$ and $v$ represent the densities of the prey and the

*This work is supported in part by the National Science Foundations of China (11271236), the Program of New Century Excellent Talents in University of Ministry of Education of China (NCET-12-0894), the Shaanxi New-star Plan of Science and Technology of China (2015KJXX-21), and also by the Natural Science Basic Research Plan in Shaanxi Province of China (2015JQ1023).

${ }^{\dagger}$ Corresponding author: jiayf@snnu.edu.cn

(C) 2016. This manuscript version is made available under the Elsevier user license http://www.elsevier.com/open-access/userlicense/1.0/ 
predator. The diffusion terms $d_{j}, j=1,2$ stand for natural dispersive force of movement of an individual, while $d_{3}$ or $d_{1} d_{3}$ describes the mutual interferences between individuals and is usually referred as the cross-diffusion pressure measuring the situation that the prey keeps away from the predator; $c$ and $d$ refer to the conversion rate of prey captured by the predator and the predator's death rate, respectively. The coefficients $c, d, d_{1}$ and $d_{2}$ are positive constants and $d_{3}$ is non-negative constant. The homogeneous Neumann boundary condition indicates that the system (1.1) is self-contained with zero population flux across the boundary. System (1.1) implies that, in addition to the natural dispersive force, the diffusion also depends on the population pressure from other species. For more biological background and significance on (1.1), one can refer to [1-7] and the references therein.

In system (1.1), the function $g(u)$ represents the growth rate of the prey in the absence of predator, $p(u)$ is called the functional response of the predator to prey and describes the change in the rate of exploitation of the prey by a predator as a result of a change in the prey density. Indeed, the growth of the predator is enhanced in the presence of the prey by an amount proportional to the number of prey. Thus, the functional response can be interpreted as the consumption rate of the prey by an individual predator. The functions $g(u) \in C^{1}([0,+\infty))$ and $p(u) \in C([0,+\infty)) \cap C^{2}((0,+\infty))$ are assumed to satisfy the following two hypotheses throughout this paper.

(H1) $g(0)>0$ and $g^{\prime}(u)<0$, for all $u \geq 0$. And there exists a unique positive constant $S$, such that $g(S)=0$;

(H2) $p(0)=0, \lim _{u \rightarrow 0^{+}} p^{\prime}(u)<\infty, c p(S)>d$, and $p^{\prime}(u)>0$ for all $u>0$.

As examples of $g(u)$ satisfying the hypothesis (H1), the following two specific forms can be introduced.

Form 1: $g(u)=\frac{r(K-u)}{K+\varepsilon u} ; \quad$ Form 2: $g(u)=r\left(1-\left(\frac{u}{K}\right)^{\theta}\right)$, where $r, K$ and $\theta \geq 1$ are positive constants and $\varepsilon$ is non-negative constant. In view of [8], Form 2 is called $\theta$-logistic growth rate. With respect to the aspect of the functional response $p(u)$, it can be classified into many types, such as

Type 1: $p(u)=u, \quad$ Type 2: $p(u)=\frac{u}{a+u}, \quad$ Type 3: $p(u)=\frac{u^{2}}{a+b u+u^{2}}$,

Type 4: $p(u)=\frac{u}{a+u^{2}}, \quad$ Type 5: $1-e^{-a u}, \quad$ Type 6: $p(u)=e^{-a u}$, where $a, b$ are positive constants. Types 1-4 are called Holling-type functional responses, a combination of Holling-types I, II, III and IV, respectively (for examples, see [9-12]). Types 5-6 are named as the Ivlev and the inverted Ivlev functional responses (see [13-15]). Note that Type 1-5 functional responses all satisfy the hypothesis (H2). The predator-prey system with $g(u)=r(K-u)$ and $p(u)=u$ is the well-known and classical Lotka-Volterra predator-prey system.

In mathematical ecology, the classical predator-prey system, contributed to Lotka and Volterra independently in the 1920s, reflects only population changes due to predation in a situation where predator and prey densities are not spatially dependent. It usually considers neither the fact that population is distributed non-homogenously, nor the fact that predators and preys naturally develop strategies for survival. Both of these considerations involve diffusion processes which can be illustrated as different concentration levels of predators and preys causing different movements. Such movements can be determined by 
the concentration of the same species (diffusion) and that of other species (cross-diffusion). Generally speaking, population growth models that include diffusion and cross-diffusion can produce more complex population dynamics than those without taking these factors into account.

For effects of cross-diffusion on the dynamics of different systems, there have been many valuable results and advances. For example, in [16-17], the authors gave rather detailed study of predator-prey systems with cross-diffusion effects. They established both the existence results of the positive steady states and the stability of these solutions. A reaction-diffusion system with cross-diffusion on bounded or unbounded domains was considered in [18], where the authors mainly studied the linear stability of the constant equilibrium. They not only showed that the cross-diffusion could destabilize a stable uniform equilibrium for kinetic and self-diffusion reaction systems, but also showed that the cross-diffusion could also stabilize an unstable uniform equilibrium for the self-diffusion reaction system (but the same uniform equilibrium is stable for the kinetic system). In [19], the authors investigated the process of pattern formation in a two dimensional domain for a reaction-diffusion system with nonlinear diffusion terms, the linear stability analysis showed that the cross-diffusion, through Turing bifurcation, is the key mechanism for the formation of spatial patterns. Paper [20] was concerned with the behavior of a competition reaction-diffusion system with nonlinear diffusion effects, that is, with cross-diffusion effects. The authors discussed the boundedness of non-constant positive solutions. Based on the boundedness of solutions, they mainly investigated the existence of the positive steady state by the monotone iteration method. There also have many valuable surveys on the mathematical developments of cross-diffusion equations arising from various research fields, such as [21-26], etc.

In system (1.1), the cross-diffusion pressure $d_{3}$ ( or $d_{1} d_{3}$ ), which can not be reflected in classical predator-prey systems, plays a central role in producing various spatial patterns in population dynamics (see [27-28] for example). We may regard $d_{1} u\left(1+d_{3} v\right)$ as living conditions of the prey, so that the diffusion is toward places of better living conditions. In system (1.1), the diffusion $d_{1} \Delta\left(\left(1+d_{3} v\right) u\right)$ of population can be understood as follows. Writing $\Delta u=\operatorname{div}(\nabla u)$, we can regard $\nabla u$ as the flux. The prey $u$ diffuses with flux

$$
-\nabla\left(d_{1} u+d_{1} d_{3} u v\right)=-d_{1} d_{3} u \nabla v-\left(d_{1}+d_{1} d_{3} v\right) \nabla u .
$$

We observe that, as $d_{1} d_{3} u>0$, the part $-d_{1} d_{3} u \nabla v$ of the flux is directed toward the decreasing population density of the predator. In particular, when $d_{3}$ is large, $-d_{1} d_{3} u \nabla v$ represents the prey running away from a large group of predators, in agreement with observed general pattern of predator-prey movement, see [28] for a more detailed discussion on relevant biological models.

Since the cross-diffusion term is introduced, the corresponding analysis in the subsequence is a bit difficult and requires more techniques than models without cross-diffusion. As a result, we, perhaps, demonstrate that the cross-diffusion may have crucial impact on the dynamics of certain predator-prey systems. In fact, our results show that if the cross-diffusion term is not very large, then the system has positive solutions.

The main purpose of this paper is to study the effect of diffusion and cross-diffusion pressures on the non-constant positive steady states of system (1.1). Specifically, we inves- 
tigate the existence and non-existence of non-constant positive solutions to the following elliptic system

$$
\begin{cases}-d_{1} \Delta\left(\left(1+d_{3} v\right) u\right)=u g(u)-p(u) v, & x \in \Omega, \\ -d_{2} \Delta v=v(-d+c p(u)), & x \in \Omega, \\ \partial_{\nu} u=\partial_{\nu} v=0, & x \in \partial \Omega .\end{cases}
$$

The paper is organized as followings. In the next section, we establish a priori upper and lower bounds for positive solutions of system (1.2) by using the maximum principle and the Harnack inequality. In Section 3, we give the non-existence of non-constant positive steady states by employing the energy integral method. In Section 4 , we first investigate the stability of constant equilibrium point of (1.2), and then we consider the existence of non-constant positive steady states for a large range of diffusion coefficients and suitable cross-diffusion coefficient by using the Leray-Schauder degree theory.

\section{A priori estimate for positive solutions}

The aim of this section is to give a priori upper and lower bounds for positive solutions of system (1.2). To this end, we first give following two preliminaries. Where, Lemma 2.1 is a immediate result of the proof of Proposition 2.2 in [21].

Lemma 2.1. (Maximum principle) If $u \in C^{2}(\Omega) \cap C^{1}(\bar{\Omega})$ satisfies $\partial_{\nu} u=0$ on $\partial \Omega$ and achieves its maximum on a point $x_{0} \in \bar{\Omega}$, then $-\Delta u\left(x_{0}\right) \geq 0$.

Lemma 2.2. (Harnack inequality) [29] Assume that $c(x) \in C(\bar{\Omega})$ and let $\omega(x) \in$ $C^{2}(\Omega) \cap C^{1}(\bar{\Omega})$ be a positive solution to

$$
\Delta \omega(x)+c(x) \omega(x)=0, x \in \Omega ; \quad \partial_{\nu} \omega=0, x \in \partial \Omega .
$$

Then there exists a positive constant $C^{*}=C^{*}\left(\|c\|_{\infty}, \Omega\right)$, such that $\max _{\bar{\Omega}} \omega \leq C^{*} \min _{\bar{\Omega}} \omega$.

Theorem 2.1. Let $D_{1}, D_{2}, D_{3}$ be given positive constants. Then there exists a positive constant $C=C\left(c, d, D_{1}, D_{2}, D_{3}, \Omega\right)$ such that every positive solution $(u, v)$ of system (1.2) satisfies $C^{-1}<u(x), v(x)<C$ for $d_{1} \geq D_{1}, d_{2} \geq D_{2}$ and $d_{3} \leq D_{3}$.

Proof. Assume that $(u, v)$ is a positive solution of system (1.2) and denote $\phi=$ $\left(1+d_{3} v\right) u$. Then system $(1.2)$ becomes

$$
\begin{cases}-d_{1} \Delta \phi=u g(u)-p(u) v, & x \in \Omega, \\ -d_{2} \Delta v=v(-d+c p(u)), & x \in \Omega, \\ \partial_{\nu} \phi=\partial_{\nu} v=0, & x \in \partial \Omega .\end{cases}
$$

Let $x_{1} \in \bar{\Omega}$ be a point such that $\phi\left(x_{1}\right)=\max _{\bar{\Omega}} \phi(x)$. Applying Lemma 2.1 to the first equation of system (2.1), we have

$$
u\left(x_{1}\right) g\left(u\left(x_{1}\right)\right)-p\left(u\left(x_{1}\right)\right) v\left(x_{1}\right) \geq 0 .
$$

Therefore $u\left(x_{1}\right) g\left(u\left(x_{1}\right)\right) \geq 0$. By (H1), we obtain $0<u\left(x_{1}\right) \leq S$ and $0<v\left(x_{1}\right) \leq$ $\frac{u\left(x_{1}\right) g\left(u\left(x_{1}\right)\right)}{p\left(u\left(x_{1}\right)\right)} \leq \frac{S g\left(u\left(x_{1}\right)\right)}{p\left(u\left(x_{1}\right)\right)} \triangleq M$ (Here, it can be shown that $\sup _{u \in(0, S)} \frac{u g(u)}{p(u)}<\infty$ by the condition $\left.\lim _{u \rightarrow 0^{+}} p^{\prime}(u)<\infty\right)$. Thus

$$
\max _{\bar{\Omega}} u(x) \leq \max _{\bar{\Omega}} \phi(x)=\left(1+d_{3} v\left(x_{1}\right)\right) u\left(x_{1}\right) \leq\left(1+d_{3} M\right) S \triangleq C_{1} .
$$


Multiplying $c$ to the first equation of (1.2) and adding it to the second equation and then integrating over $\Omega$, we obtain

$$
\int_{\Omega}\left(-c d_{1} \Delta\left(\left(1+d_{3} v\right) u\right)-d_{2} \Delta v\right) d x=\int_{\Omega}(-d v+c u g(u)) d x .
$$

By the divergence theorem, we know that $\int_{\Omega}\left(-c d_{1} \Delta\left(\left(1+d_{3} v\right) u\right)-d_{2} \Delta v\right) d x=0$. So

$$
d \int_{\Omega} v d x=\int_{\Omega} c u g(u) d x \leq \int_{\Omega} c g(0) C_{1} d x=c g(0) C_{1}|\Omega|,
$$

that is, $\int_{\Omega} v d x \leq \frac{c g(0) C_{1}|\Omega|}{d}$.

On the other hand, system (1.2) can also be written as

$$
\begin{cases}-\Delta \phi=\frac{g(u)-\frac{p(u)}{u} v}{d_{1}\left(1+d_{3} v\right)} \phi, & x \in \Omega, \\ -\Delta v=\frac{v(-d+c p(u))}{d_{2}}, & x \in \Omega, \\ \partial_{\nu} \phi=\partial_{\nu} v=0, & x \in \partial \Omega .\end{cases}
$$

As $\frac{-d+c p(u)}{d_{2}}<\frac{c p(S)}{D_{2}}<\infty$ for $d_{2} \geq D_{2}$ and $u<S$, Lemma 2.2 holds for $v$, so

$$
\max _{\bar{\Omega}} v \leq C_{0}^{*} \min _{\bar{\Omega}} v
$$

for some positive constant $C_{0}^{*}$. Therefore, we obtain

$$
\max _{\bar{\Omega}} v \leq C_{0}^{*} \min _{\bar{\Omega}} v \leq \frac{C_{0}^{*} \int_{\Omega} v d x}{|\Omega|} \leq \frac{c g(0) C_{1} C_{0}^{*}}{d} \triangleq C_{2}
$$

By integrating the first equation of system (1.2) over $\Omega$, we have

$$
\int_{\Omega}(u g(u)-p(u) v) d x=0 .
$$

Thus, there exists a point $x_{2} \in \Omega$ such that

$$
u\left(x_{2}\right) g\left(u\left(x_{2}\right)\right)-p\left(u\left(x_{2}\right)\right) v\left(x_{2}\right)=0 .
$$

By assumptions (H1) and (H2), we know $0<u\left(x_{2}\right)<S$. Then $v\left(x_{2}\right)=\frac{u\left(x_{2}\right) g\left(u\left(x_{2}\right)\right)}{p\left(u\left(x_{2}\right)\right)}>0$. If $\min _{\bar{\Omega}} v=0$, then (2.2) implies $\max _{\bar{\Omega}} v=0$. So $v \equiv 0$ uniformly in $\Omega$. This is a contradiction, and $v$ has a positive lower bound for $d_{2} \geq D_{2}$.

In the following, we show that $u$ has a positive lower bound.

Since $p(u) \in C^{2}((0,+\infty))$ and $\lim _{u \rightarrow 0^{+}} \frac{p(u)}{u}=\lim _{u \rightarrow 0^{+}} p^{\prime}(u)<\infty$, then there exists a positive constant $\bar{p}$ such that $\frac{p(u)}{u} \leq \bar{p}$ for small $0<u \leq S$. Clearly, we have $\frac{g(u)-\frac{p(u) v}{u}}{d_{1}\left(1+d_{3} v\right)} \leq$ $\frac{g(0)+\bar{p} C_{2}}{D_{1}}<\infty$ when $d_{1} \geq D_{1}$. Thus Lemma 2.2 holds for $\phi$, that is $\max _{\bar{\Omega}} \phi \leq C_{1}^{*} \min _{\bar{\Omega}} \phi$ for some positive constant $C_{1}^{*}$. Hence, we get

$$
\frac{\max _{\bar{\Omega}} u}{\min _{\bar{\Omega}} u} \leq \frac{\max _{\bar{\Omega}} \phi}{\min _{\bar{\Omega}} \phi} \cdot \frac{1+d_{3} \max _{\bar{\Omega}} v}{1+d_{3} \min _{\bar{\Omega}} v} \leq C_{1}^{*}\left(1+d_{3} \max _{\bar{\Omega}} v\right) \leq C_{1}^{*}\left(1+d_{3} C_{2}\right) \triangleq C_{2}^{*} .
$$


Assume, on the contrary, that there exists a sequence $\left\{\left(d_{1 n}, d_{2 n}, d_{3 n}\right)\right\}, n=1,2, \cdots$, satisfying $d_{1 n} \geq D_{1}, d_{2 n} \geq D_{2}$, and $d_{3 n} \leq D_{3}$ for some $D_{3}>0$, such that the corresponding positive solutions $\left(u_{n}, v_{n}\right)$ of system $(1.2)$ with $\left(d_{1}, d_{2}, d_{3}\right)=\left(d_{1 n}, d_{2 n}, d_{3 n}\right)$ satisfy $\min _{\bar{\Omega}} u_{n} \rightarrow 0$ as $n \rightarrow \infty$. Combining with (2.3), we have $\max _{\bar{\Omega}} u_{n} \rightarrow 0$ as $n \rightarrow \infty$. By the regularity theory for elliptic equations in [30], we know that there exists a subsequence of $\left\{\left(u_{n}, v_{n}\right)\right\}$, which will be also denoted by $\left\{\left(u_{n}, v_{n}\right)\right\}$, such that $u_{n} \rightarrow 0$ uniformly as $n \rightarrow \infty$. Integrating the second equation of system $(1.2)$ with $(u, v)=\left(u_{n}, v_{n}\right)$, we obtain

$$
\int_{\Omega} v_{n}\left(-d+c p\left(u_{n}\right)\right) d x=0 .
$$

Since $u_{n} \rightarrow 0$ as $n \rightarrow \infty$, we have $-d+c p\left(u_{n}\right)<0$ in $\bar{\Omega}$ for any large $n$. This contradicts the integrating identity $(2.4)$ and the fact $v_{n}>0$. The proof is completed.

\section{Non-existence of positive solution}

In this section, we obtain the results of non-existence of positive solution to system (1.2) by using the energy integral method. The first non-existence result is characterized directly by the function $p(u)$.

Theorem 3.1. If $p(S) \leq \frac{d}{c}$, then system (1.2) has no non-constant positive solution.

Proof. Since $g(u)<0$ for $u \geq S$, we only need to consider the case $u<S$. Suppose on the contrary that (1.2) has a non-constant positive solution $(u, v)$ for $p(S) \leq \frac{d}{c}$. Then $v$ must be non-constant, otherwise, it is easily seen that $u$ must be constant from the second equation of (1.2). Using the fact that $p(u)$ is increasing in $u$, and integrating the second equation of (1.2) over $\Omega$, we get

$$
0=-d_{2} \int_{\Omega} \Delta v d x=\int_{\Omega} v(c p(u)-d) d x<\int_{\Omega} v(c p(S)-d) d x .
$$

Since $v>0$, we must have $p(S)>\frac{d}{c}$. This contradiction completes the proof. Therefore, if (1.2) has a positive solution, it is necessary that $p(S)>\frac{d}{c}$. This is just one of the conditions in (H2) which $p(u)$ should satisfy.

Let $\lambda_{1}$ be the smallest positive eigenvalue of $-\Delta$ with homogeneous Neumann boundary condition. Set $\bar{u}=\frac{1}{|\Omega|} \int_{\Omega} u d x$ and $\bar{v}=\frac{1}{|\Omega|} \int_{\Omega} v d x$. The next non-existence result is given by considering the self- and cross-diffusion rates.

Theorem 3.2. Let

$$
\tilde{d}_{1}=\lambda_{1}+\lambda_{1}^{-1}\left(g(0)+c \lambda_{1} K_{0}\right) \quad \text { and } \quad \tilde{d}_{2}=\lambda_{1}^{-1}\left(-d+c p\left(C_{1}\right)+\frac{c K_{0}+\left(d_{1} d_{3} C_{1}\right)^{2}}{4}\right)
$$

with $K_{0}=\sup _{\bar{\Omega}} \bar{v} p^{\prime}(u)$. Then system (1.2) has no non-constant positive solution when $d_{1}, d_{2}$ and $d_{3}$ satisfy $d_{1} \geq \tilde{d}_{1}, d_{2} \geq \tilde{d}_{2}$.

Proof. Let $(u, v)$ be a positive solution of system (1.2). Multiplying the equations of 
(1.2) by $u-\bar{u}, v-\bar{v}$ respectively, and then integrating over $\Omega$, we have

$$
\begin{aligned}
& \int_{\Omega}\left[d_{1}\left(1+d_{3} v\right)|\nabla u|^{2}+d_{2}|\nabla v|^{2}+d_{1} d_{3} u \nabla u \nabla v\right] d x \\
= & \int_{\Omega}[(u-\bar{u})(u g(u)-p(u) v)+(v-\bar{v}) v(-d+c p(u))] d x \\
= & \int_{\Omega}(u-\bar{u})[g(u)(u-\bar{u})+\bar{u}(g(u)-g(\bar{u}))-p(u)(v-\bar{v})-\bar{v}(p(u)-p(\bar{u}))] d x \\
& +\int_{\Omega}(v-\bar{v})[-d(v-\bar{v})+c p(u)(v-\bar{v})+c \bar{v}(p(u)-p(\bar{u}))] d x \\
= & \left.\int_{\Omega}(u-\bar{u})\left[g(u)(u-\bar{u})+\bar{u} g^{\prime}(\xi)(u-\bar{u})-p(u)(v-\bar{v})-\bar{v} p^{\prime}(\eta)(u-\bar{u})\right)\right] d x \\
& +\int_{\Omega}(v-\bar{v})\left[-d(v-\bar{v})+c p(u)(v-\bar{v})+c \bar{v}(u-\bar{u}) p^{\prime}(\zeta)\right] d x \\
= & \int_{\Omega}\left[(u-\bar{u})^{2}\left(g(u)+\bar{u} g^{\prime}(\xi)-\bar{v} p^{\prime}(\eta)\right)\right. \\
& \left.+(v-\bar{v})^{2}(-d+c p(u))+(u-\bar{u})(v-\bar{v})\left(-p(u)+c \bar{v} p^{\prime}(\zeta)\right)\right] d x \\
< & \int_{\Omega}\left[(u-\bar{u})^{2} g(0)+(v-\bar{v})^{2}\left(-d+c p\left(C_{1}\right)\right)+c K_{0}|u-\bar{u} \| v-\bar{v}|\right] d x,
\end{aligned}
$$

where $0<\xi, \eta, \zeta \leq C_{1}$ and $K_{0}=\sup _{\bar{\Omega}} \bar{v} p^{\prime}(u)$ (Here we note that $p^{\prime}(u)$ is bounded in any finite interval in view of the assumptions $p(u) \in C^{2}((0,+\infty))$ and H2). By Theorem 2.1 and the Cauchy inequality, we have

$$
\begin{aligned}
& \int_{\Omega}\left[d_{1}\left(1+d_{3} v\right)|\nabla u|^{2}+d_{2}|\nabla v|^{2}\right] d x \\
< & \int_{\Omega}\left[(u-\bar{u})^{2}\left(g(0)+c K_{0} K\right)+(v-\bar{v})^{2}\left(-d+c p\left(C_{1}\right)+\frac{c K_{0}}{4 K}\right)\right. \\
& \left.+K|\nabla u|^{2}+\frac{d_{1}^{2} d_{3}^{2} u^{2}}{4 K}|\nabla v|^{2}\right] d x
\end{aligned}
$$

with $K$ be any positive constant. Then using the Poincaré inequality, we get

$$
\begin{aligned}
& \int_{\Omega}\left[d_{1}\left(1+d_{3} v\right)|\nabla u|^{2}+d_{2}|\nabla v|^{2}\right] d x \\
< & \int_{\Omega}\left[\left(\lambda_{1}+\lambda_{1}^{-1}\left(g(0)+c \lambda_{1} K_{0}\right)\right)|\nabla u|^{2}\right. \\
& \left.+\left(\lambda_{1}^{-1}\left(-d+c p\left(C_{1}\right)+\frac{c K_{0}+\left(d_{1} d_{3} C_{1}\right)^{2}}{4}\right)\right)|\nabla v|^{2}\right] d x
\end{aligned}
$$

by taking $K=\lambda_{1}$. Hence, we know that (1.2) has no non-constant positive solution with the assumptions.

Remark 3.1. If $d_{3}$ is small, say $d_{3} \leq \tilde{d}_{3}$ for some positive constant $\tilde{d}_{3}$, then Theorem 3.2 implies that system (1.2) does not permit non-constant positive solution for large $d_{1}$ and $d_{2}$. This is consistent with the non-existence results in those classical Gause-type predator-prey models. 


\section{Stability of constant positive solution and existence of non-constant positive solutions}

This section is devoted to some aspects of positive solutions to system (1.2), including the stability of the unique constant positive equilibrium point and the existence of nonconstant positive solutions.

\subsection{Stability of constant solutions}

In this subsection, we start with the analysis on the local stability of constant solutions to system (1.2). And then, we will investigate the global stability of constant solutions. By computation, we know that (1.1), and so (1.2), has a unique constant solution $U^{*}=\left(u^{*}, v^{*}\right)$ under the hypotheses (H1) and (H2), where

$$
u^{*}=p^{-1}\left(\frac{d}{c}\right), \quad v^{*}=\frac{u^{*} g\left(u^{*}\right)}{p\left(u^{*}\right)}=\frac{c p^{-1}\left(\frac{d}{c}\right) g\left(p^{-1}\left(\frac{d}{c}\right)\right)}{d}
$$

with $p^{-1}$ be the inverse of $p$.

The local stability of $U^{*}$ reads as follow.

Theorem 4.1. $U^{*}$ is locally asymptotically stable if $v^{*} p^{\prime}\left(u^{*}\right)>g\left(u^{*}\right)+u^{*} g^{\prime}\left(u^{*}\right)$, and unstable if $v^{*} p^{\prime}\left(u^{*}\right)<g\left(u^{*}\right)+u^{*} g^{\prime}\left(u^{*}\right)$.

Proof. Denote $L$ by the $2 \times 2$ matrix operator

$$
L=\left(\begin{array}{cc}
-d_{1} \Delta-g\left(u^{*}\right)-u^{*} g^{\prime}\left(u^{*}\right)+v^{*} p^{\prime}\left(u^{*}\right) & p\left(u^{*}\right) \\
-c v^{*} p^{\prime}\left(u^{*}\right) & -d_{2} \Delta
\end{array}\right) .
$$

Here, we note that $L$ is the linearized operator of (1.2) at $U^{*}$. Let $\left\{\lambda_{i}\right\}_{i=0}^{\infty}$ be the sequence of eigenvalues for $-\Delta$ on $\Omega$ with homogeneous Neumann boundary condition and $X_{i}$ be the eigenspace corresponding to each $\lambda_{i}$. Then for each $i=0,1,2, \cdots$, we know that $X_{i}$ is invariant under the operator $L$. Furthermore, it is easy to check that $\mu$ is an eigenvalue of $L$ on some $X_{i}$ if and only if $\mu$ is an eigenvalue of the matrix

$$
L_{i} \triangleq\left(\begin{array}{cc}
d_{1} \lambda_{i}-g\left(u^{*}\right)-u^{*} g^{\prime}\left(u^{*}\right)+v^{*} p^{\prime}\left(u^{*}\right) & p\left(u^{*}\right) \\
-c v^{*} p^{\prime}\left(u^{*}\right) & d_{2} \lambda_{i}
\end{array}\right)
$$

The determinant $\operatorname{det} L_{i}$ and the trace $\operatorname{tr} L_{i}$ of $L_{i}$ are

$$
\operatorname{det} L_{i}=d_{1} d_{2} \lambda_{i}^{2}+d_{2} \lambda_{i}\left(v^{*} p^{\prime}\left(u^{*}\right)-\left(g\left(u^{*}\right)+u^{*} g^{\prime}\left(u^{*}\right)\right)\right)+c v^{*} p\left(u^{*}\right) p^{\prime}\left(u^{*}\right)
$$

and

$$
\operatorname{tr} L_{i}=\left(d_{1}+d_{2}\right) \lambda_{i}+v^{*} p^{\prime}\left(u^{*}\right)-\left(g\left(u^{*}\right)+u^{*} g^{\prime}\left(u^{*}\right)\right),
$$

respectively. It is clear that $\operatorname{det} L_{i}>0$ and $\operatorname{tr} L_{i}>0$ for all $i$ provided that $v^{*} p^{\prime}\left(u^{*}\right)>$ $g\left(u^{*}\right)+u^{*} g^{\prime}\left(u^{*}\right)$. In this case, the two eigenvalues $\mu_{i}^{+}$and $\mu_{i}^{-}$of $L_{i}$ both have positive real parts. Hence $U^{*}$ is locally asymptotically stable for system (1.2). On the other hand, if $v^{*} p^{\prime}\left(u^{*}\right)<g\left(u^{*}\right)+u^{*} g^{\prime}\left(u^{*}\right)$, then we have $\operatorname{tr} L_{0}<0$. This shows that $L_{0}$ has at least an eigenvalue with negative real part, and therefore $U^{*}$ is unstable. 
Now, we consider the global stability of $U^{*}$.

Theorem 4.2. $U^{*}$ is globally asymptotically stable if $p\left(u^{*}\right)>C^{2} g(0)$ with $C$ being given in Theorem 2.1.

Proof. We discuss the global stability of $U^{*}$ by Lyapunov procedure. Define

$$
L(u(x, t), v(x, t))=\int_{\Omega} \int_{u^{*}}^{u} \frac{c p(\xi)-c p\left(u^{*}\right)}{p(\xi)} d \xi d x+\int_{\Omega} \int_{v^{*}}^{v} \frac{\eta-v^{*}}{\eta} d \eta d x .
$$

Then

$$
\begin{aligned}
L^{\prime}(t)= & c \int_{\Omega} \frac{\left(p(u)-p\left(u^{*}\right)\right) u_{t}}{p(u)} d x+\int_{\Omega} \frac{\left(v-v^{*}\right) v_{t}}{v} d x \\
= & c \int_{\Omega}\left(1-\frac{p\left(u^{*}\right)}{p(u)}\right)\left(d_{1} \Delta\left(\left(1+d_{3} v\right) u\right)+u g(u)-p(u) v\right) d x \\
& +\int_{\Omega}\left(1-\frac{v^{*}}{v}\right)\left(d_{2} \Delta v+v(-d+c p(u))\right) d x \\
= & c d_{1} \int_{\Omega}\left(1-\frac{p\left(u^{*}\right)}{p(u)}\right) \Delta\left(\left(1+d_{3} v\right) u\right) d x-c \int_{\Omega}\left(p(u)-p\left(u^{*}\right)\right)\left(v-\frac{u g(u)}{p(u)}\right) d x \\
& +d_{2} \int_{\Omega}\left(1-\frac{v^{*}}{v}\right) \Delta v d x-\int_{\Omega}(d-c p(u))\left(v-v^{*}\right) d x \\
= & c d_{1} \int_{\Omega}\left(1-\frac{p\left(u^{*}\right)}{p(u)}\right) \Delta\left(\left(1+d_{3} v\right) u\right) d x+d_{2} \int_{\Omega}\left(1-\frac{v^{*}}{v}\right) \Delta v d x \\
& +c \int_{\Omega} u g(u)\left(1-\frac{p\left(u^{*}\right)}{p(u)}\right) d x-\int_{\Omega}(c p(u)-d) v^{*} d x \\
= & c d_{1} \int_{\Omega}\left(1-\frac{p\left(u^{*}\right)}{p(u)}\right) \Delta\left(\left(1+d_{3} v\right) u\right) d x+c \int_{\Omega} u g(u)\left(1-\frac{p\left(u^{*}\right)}{p(u)}\right) d x .
\end{aligned}
$$

Further computation gives that

$$
\begin{aligned}
& c d_{1} \int_{\Omega}\left(1-\frac{p\left(u^{*}\right)}{p(u)}\right) \Delta\left(\left(1+d_{3} v\right) u\right) d x \\
= & c d_{1} \int_{\Omega}\left(1-\frac{p\left(u^{*}\right)}{p(u)}\right) \Delta u d x+c d_{1} d_{3} \int_{\Omega}\left(1-\frac{p\left(u^{*}\right)}{p(u)}\right) v \Delta u d x \\
& \left.+2 c d_{1} d_{3} \int_{\Omega}\left(1-\frac{p\left(u^{*}\right)}{p(u)}\right) \nabla u \nabla v\right) d x+c d_{1} d_{3} \int_{\Omega}\left(1-\frac{p\left(u^{*}\right)}{p(u)}\right) u \Delta v d x \\
= & -c d_{1} p\left(u^{*}\right) \int_{\Omega} \frac{p^{\prime}(u)|\nabla u|^{2}}{p^{2}(u)} d x+c d_{1} d_{3} p\left(u^{*}\right) \int_{\Omega} \frac{\left(p(u) \nabla v-v p^{\prime}(u) \nabla u\right) \nabla u}{p^{2}(u)} d x \\
& -2 c d_{1} d_{3} p\left(u^{*}\right) \int_{\Omega} \frac{\nabla u \nabla v}{p(u)} d x+c d_{1} d_{3} p\left(u^{*}\right) \int_{\Omega} \frac{\left(p(u)-u p^{\prime}(u)\right) \nabla u \nabla v}{p^{2}(u)} d x \\
= & -c d_{1} p\left(u^{*}\right) \int_{\Omega} \frac{p^{\prime}(u)|\nabla u|^{2}}{p^{2}(u)} d x-c d_{1} d_{3} p\left(u^{*}\right) \int_{\Omega} \frac{v p^{\prime}(u)|\nabla u|^{2}}{p^{2}(u)} d x \\
& -c d_{1} d_{3} p\left(u^{*}\right) \int_{\Omega} \frac{u p^{\prime}(u) \nabla u \nabla v}{p^{2}(u)} d x
\end{aligned}
$$




$$
\begin{aligned}
& =-c d_{1} p\left(u^{*}\right) \int_{\Omega} \frac{p^{\prime}(u) \nabla u}{p^{2}(u)}\left(\left(1+d_{3} v\right) \nabla u+d_{3} u \nabla v\right) d x \\
& =c d_{1} p\left(u^{*}\right) \int_{\Omega}\left(\left(1+d_{3} v\right) \nabla u+d_{3} u \nabla v\right) d\left(\frac{1}{p(u)}\right) \\
& =-c d_{1} p\left(u^{*}\right) \int_{\Omega} \frac{1}{p(u)} d\left(\nabla\left(1+d_{3} v\right) u\right) d x \\
& =c p\left(u^{*}\right) \int_{\Omega} \frac{u g(u)-p(u) v}{p(u)} d x .
\end{aligned}
$$

Therefore, we have

$$
\begin{aligned}
L^{\prime}(t) & =c p\left(u^{*}\right) \int_{\Omega} \frac{u g(u)-p(u) v}{p(u)} d x+c \int_{\Omega} u g(u)\left(1-\frac{p\left(u^{*}\right)}{p(u)}\right) d x \\
& =c \int_{\Omega}\left(u g(u)-p\left(u^{*}\right) v\right) d x .
\end{aligned}
$$

Since $g(u) \leq g(0)$, combining this fact with Theorem 2.1, we know that $L^{\prime}(t)<0$ provided that $p\left(u^{*}\right)>C^{2} g(0)$. This completes the proof.

\subsection{Existence of non-constant positive solutions}

Now, we investigate the existence of non-constant positive solutions to system (1.2) for suitable diffusion coefficients as parameters. Specifically, we consider the cases that the self-diffusion is large and the cross-diffusion is small. The main tool used here in proving the existence of non-constant positive solutions is the Leray-Schauder degree theory which has been widely used in different aspects.

For convenience, we need to introduce some notations.

Denote

$$
X=\left\{(u, v) \in\left(C^{2}(\Omega) \cap C^{1}(\bar{\Omega})\right)^{2} \mid \partial_{\nu} u=\partial_{\nu} v=0 \text { on } \partial \Omega\right\} .
$$

Then we have the decomposition $X=\oplus_{i=0}^{\infty} X_{i}$ with $X_{i}$ being the eigenspace corresponding to each $\lambda_{i}$. Set

$$
\begin{gathered}
D=\left(d_{1}, d_{2}, d_{3}\right), \\
B(C)=\left\{(u, v) \in X \mid C^{-1}<u, v<C\right\}, \\
U=(u, v), \quad \Phi(U)=\left(d_{1}\left(1+d_{3} v\right) u, d_{2} v\right)
\end{gathered}
$$

and

$$
G(U)=(u g(u)-p(u) v, v(-d+c p(u))) .
$$

Then it is easy to see that $\operatorname{det} \Phi_{U}(U)>0$ for all non-negative $(u, v)$, so $\Phi_{U}^{-1}$ exists. Thus, we can rewrite (1.2) as

$$
F(D ; U) \triangleq U-(I-\Delta)^{-1}\left\{\Phi_{U}^{-1}(U)[G(U)+\mathfrak{S}(U)+U\}=0\right.
$$

with $\mathfrak{S}(U)=\left(2 d_{1} d_{3} \nabla u \nabla v, 0\right)$, where $I$ is the identity and $(I-\Delta)^{-1}$ is the inverse of $I-\Delta$ on $X$ under the homogeneous Neumann boundary condition. As $F(D ; \cdot)$ a compact 
perturbation of the identity operator, the Leray-Schauder degree $\operatorname{deg}(F(D ; \cdot), 0, B)$ is welldefined if $F(D ; U) \neq 0$ for all $U \in \partial B$.

The linearizition of $F(D ; U)$ at $U^{*}$ is

$$
D_{U} F\left(D ; U^{*}\right)=I-(I-\Delta)^{-1}\left[\Phi_{U}^{-1}\left(U^{*}\right) G_{U}\left(U^{*}\right)+I\right] .
$$

It is easy to show that $\lambda$ is an eigenvalue of $D_{U} F\left(D ; U^{*}\right)$ on $X_{i}$ if and only if $\lambda$ is an eigenvalue of the matrix

$$
I-\frac{1}{1+\lambda_{i}}\left[\Phi_{U}^{-1}\left(U^{*}\right) G_{U}\left(U^{*}\right)+I\right]=\frac{1}{1+\lambda_{i}}\left[\lambda_{i} I-\Phi_{U}^{-1}\left(U^{*}\right) G_{U}\left(U^{*}\right)\right] .
$$

Let

$$
H\left(D, U^{*} ; \lambda_{i}\right)=\operatorname{det}\left[\lambda_{i} I-\Phi_{U}^{-1}\left(U^{*}\right) G_{U}\left(U^{*}\right)\right] .
$$

Then, the number of eigenvalues with negative real parts of $D_{U} F\left(D ; U^{*}\right)$ on $X_{i}$ is odd if and only if

$$
H\left(D, U^{*} ; \lambda_{i}\right)<0 .
$$

If $H\left(D, U^{*} ; \lambda_{i}\right) \neq 0$ for all integer $i \geq 0$, then 0 is not an eigenvalue of $D_{U} F\left(D ; U^{*}\right)$. This implies that $D_{U} F\left(D ; U^{*}\right)$ is a homeomorphism from $X$ to $X$. Then the implicit theorem shows that $U=U^{*}$ is an isolated solution of $F(D ; U)=0$. By the Leray-Schauder degree theory, in conclusion, we can assert the following result.

Lemma 4.1. Assume that $H\left(D, U^{*} ; \lambda_{i}\right) \neq 0$ for all $i \geq 0$. Then

$$
\operatorname{index}\left(F(D ; \cdot), U^{*}\right)=(-1)^{\sigma}, \quad \sigma=\sum_{i \geq 0, H\left(D, U^{*} ; \lambda_{i}\right)<0} m\left(\lambda_{i}\right)
$$

where $m\left(\lambda_{i}\right)$ is the algebraic multiplicity of $\lambda_{i}$.

A direct computation gives

$$
G_{U}\left(U^{*}\right)=\left(\begin{array}{cc}
g\left(u^{*}\right)+u^{*} g^{\prime}\left(u^{*}\right)-p^{\prime}\left(u^{*}\right) v^{*} & -p\left(u^{*}\right) \\
c v^{*} p^{\prime}\left(u^{*}\right) & 0
\end{array}\right) \triangleq\left(\begin{array}{cc}
A & -p\left(u^{*}\right) \\
c v^{*} p^{\prime}\left(u^{*}\right) & 0
\end{array}\right),
$$

and

$$
\Phi_{U}\left(U^{*}\right)=\left(\begin{array}{cc}
d_{1}\left(1+d_{3} v^{*}\right) & d_{1} d_{3} u^{*} \\
0 & d_{2}
\end{array}\right)
$$

Therefore, we have

$$
H\left(D, U^{*} ; \lambda\right)=\lambda^{2}-\left(\frac{A}{d_{1}\left(1+d_{3} v^{*}\right)}+\frac{d_{3} u^{*} p\left(u^{*}\right)}{d_{2}\left(1+d_{3} v^{*}\right)}\right) \lambda+\frac{c v^{*} p\left(u^{*}\right) p^{\prime}\left(u^{*}\right)}{d_{1} d_{2}\left(1+d_{3} v^{*}\right)} .
$$

Note that $\frac{c v^{*} p\left(u^{*}\right) p^{\prime}\left(u^{*}\right)}{d_{1} d_{2}\left(1+d_{3} v^{*}\right)}>0$. We will restrict our attention to large $d_{j}, j=1,2$. Note that

$$
\lim _{d_{j} \rightarrow \infty} H\left(D, U^{*} ; \lambda\right)=\lambda^{2}-\Pi_{j} \lambda, j=1,2,
$$

where

$$
\Pi_{1}=\frac{d_{3} u^{*} p\left(u^{*}\right)}{d_{2}\left(1+d_{3} v^{*}\right)}, \quad \Pi_{2}=\frac{A}{d_{1}\left(1+d_{3} v^{*}\right)} .
$$


In order to present the existence result, we need the following lemma.

Lemma 4.2. Let $c, d$ be given such that $A>0$. If $d_{1} \geq d_{1}^{*}, d_{2} \geq d_{2}^{*}$ and $d_{3} \leq d_{3}^{*}$ for some positive constants $d_{1}^{*}, d_{2}^{*}$ and $d_{3}^{*}$, then

$$
\operatorname{index}\left(F(D ; \cdot), U^{*}\right)=1 \text {. }
$$

Proof. Denote

$$
H\left(D, U^{*} ; \lambda\right)=\lambda^{2}-A_{1} \lambda+A_{2}
$$

with

$$
A_{1}=\frac{A}{d_{1}\left(1+d_{3} v^{*}\right)}+\frac{d_{3} u^{*} p\left(u^{*}\right)}{d_{2}\left(1+d_{3} v^{*}\right)}, \quad A_{2}=\frac{c v^{*} p\left(u^{*}\right) p^{\prime}\left(u^{*}\right)}{d_{1} d_{2}\left(1+d_{3} v^{*}\right)} .
$$

Solve the equation $H\left(D, U^{*} ; \lambda\right)=0$. We obtain

(i) $\lambda^{ \pm}=\frac{A_{1} \pm \sqrt{A_{1}^{2}-4 A_{2}}}{2}$ if $A_{1}^{2}-4 A_{2} \geq 0$; (ii) $\lambda^{ \pm}=\frac{A_{1} \pm \mathbf{i} \sqrt{4 A_{2}-A_{1}^{2}}}{2}$ if $A_{1}^{2}-4 A_{2}<0$, where $\mathbf{i}$ is the imaginary unit. Clearly, there exist positive constants $d_{1}^{*}, d_{2}^{*}$ large enough, and $d_{3}^{*}$ small such that $\operatorname{Re} \lambda^{+}<\lambda_{1}$ when $d_{1} \geq d_{1}^{*}, d_{2} \geq d_{2}^{*}$ and $d_{3} \leq d_{3}^{*}$, where $\operatorname{Re} \lambda^{+}$is the real part of the root of $H\left(D, U^{*} ; \lambda\right)=0$ with the maximal real part. Therefore, we have $H\left(D, U^{*} ; \lambda_{i}\right)>0$ for each integer $i>0$ from above proof. Moreover,

$$
H\left(D, U^{*} ; \lambda_{0}\right)=H\left(D, U^{*} ; 0\right)=\frac{c v^{*} p\left(u^{*}\right) p^{\prime}\left(u^{*}\right)}{d_{1} d_{2}\left(1+d_{3} v^{*}\right)}>0 .
$$

This implies that the number of eigenvalues with negative real part of $D_{U} F\left(D ; U^{*}\right)$ on $X_{i}$ is not odd. Then the result follows.

Theorem 4.3. Suppose that $A>0$ for given $c, d$.

(i) Assume that $d_{2}, d_{3}$ are given such that $\Pi_{1} \in\left(\lambda_{i}, \lambda_{i+1}\right)$ for some integer $i$ and $\sigma=\sum_{k=1}^{i} m\left(\lambda_{k}\right)$ is odd. Then there exists a positive constant $d_{1}^{*}$ such that (1.2) has at least one non-constant positive solution when $d_{1} \geq d_{1}^{*}$.

(ii) Assume that $d_{1}, d_{3}$ are given such that $\Pi_{2} \in\left(\lambda_{j}, \lambda_{j+1}\right)$ for some integer $j$ and $\sigma=\sum_{k=1}^{j} m\left(\lambda_{k}\right)$ is odd. Then there exists a positive constant $d_{2}^{*}$ such that (1.2) has at least one non-constant positive solution when $d_{2} \geq d_{2}^{*}$.

(iii) Take $d_{1}, d_{2}$ such that $\operatorname{Re} \lambda^{-} \in\left(\lambda_{i}, \lambda_{i+1}\right)$ and $\operatorname{Re} \lambda^{+} \in\left(\lambda_{j}, \lambda_{j+1}\right)$ for some integers $i$ and $j$. If $\sigma=\sum_{k=i+1}^{j} m\left(\lambda_{k}\right)$ is odd, then (1.2) has at least one non-constant positive solution when $d_{3} \leq d_{3}^{*}$.

Proof. We only prove the first assertion since the second and the third cases can be showed similarly. From Remark 3.1 and Lemma 4.2, we know that there exist positive constants $d_{1}^{*}, d_{2}^{*}$ and $d_{3}^{*}$ such that $(1.2)$ does not have non-constant positive solution and $\operatorname{index}\left(F(D ; \cdot), U^{*}\right)=1$ when $d_{1} \geq d_{1}^{*}, d_{2} \geq d_{2}^{*}$ and $d_{3} \leq d_{3}^{*}$. So, $\operatorname{deg}\left(F\left(D^{*} ; \cdot\right), 0, B\right)=1$ if we take $D^{*}=\left(d_{1}^{*}, d_{2}^{*}, d_{3}^{*}\right)$ particularly. For $d_{1} \geq d_{1}^{*}, d_{2}>d_{2}^{*}$, we define a homotopy as

$$
\begin{cases}-\Delta\left(\left(t d_{1}+(1-t) d_{1}^{*}+t d_{1} d_{3} v\right) u\right)=u g(u)-p(u) v, & x \in \Omega, \\ -\Delta\left(\left(t d_{2}+(1-t) d_{2}^{*}\right) v\right)=v(-d+c p(u)), & x \in \Omega, \\ \partial_{\nu} u=\partial_{\nu} v=0, & x \in \partial \Omega,\end{cases}
$$

with $t \in[0,1]$. Then $U$ is a non-constant positive solution of (4.2) if and only if $U$ is a non-constant positive solution of the problem

$$
\widetilde{F}(t, D ; U) \triangleq U-(I-\Delta)^{-1}\left\{\widetilde{\Phi}_{U}^{-1}(U, t)\left[G(U)+\nabla U \widetilde{\Phi}_{U U}(U, t) \nabla U\right]+U\right\}=0
$$


on $X$, where $\widetilde{\Phi}(U, t)=\left(t d_{1} u+(1-t) d_{1}^{*} u+t d_{1} d_{3} u v, t d_{2} v+(1-t) d_{2}^{*} v\right)$. By Theorem 2.1, we know that all the positive solutions $(u, v)$ of $(1.2)$ are in $B(C)$ for large enough constant $C$. Thus, for all $t \in[0,1],(4.2)$ has no solution on $\partial B$, and $\operatorname{deg}(\widetilde{F}(t, D ; \cdot), 0, B)$ is well defined. By the homotopy invariance of the Leray-Schauder degree, we have

$$
\operatorname{deg}(\widetilde{F}(0, D ; \cdot), 0, B)=\operatorname{deg}(\widetilde{F}(1, D ; \cdot), 0, B) .
$$

Note that

$$
\Phi(U)=\widetilde{\Phi}(U, 1), \quad F(D ; U)=\widetilde{F}(1, D ; U), \quad F\left(D^{*} ; U\right)=\widetilde{F}(0, D ; U) .
$$

So,

$$
\begin{aligned}
\operatorname{deg}(\widetilde{F}(0, D ; \cdot), 0, B) & =\operatorname{deg}\left(F\left(D^{*} ; \cdot\right), 0, B\right), \\
\operatorname{deg}(\widetilde{F}(1, D ; \cdot), 0, B) & =\operatorname{deg}(F(D ; \cdot), 0, B) .
\end{aligned}
$$

Moreover, we have shown

$$
\operatorname{deg}\left(F\left(D^{*} ; \cdot\right), 0, B\right)=1
$$

Therefore, in this case, we get

$$
\operatorname{deg}(F(D ; \cdot), 0, B)=\operatorname{deg}\left(F\left(D^{*} ; \cdot\right), 0, B\right)=1 .
$$

From (4.1), we see that

$$
\lim _{d_{1} \rightarrow \infty} \operatorname{Re} \lambda^{-}=0, \quad \lim _{d_{1} \rightarrow \infty} \operatorname{Re} \lambda^{+}=\Pi_{1} .
$$

If $\Pi_{1} \in\left(\lambda_{i}, \lambda_{i+1}\right)$ for some integer $i$ and $d_{1}$ is large enough, we have

$$
0=\lambda_{0}<\operatorname{Re} \lambda^{-}<\lambda_{1}, \quad \operatorname{Re} \lambda^{+} \in\left(\lambda_{i}, \lambda_{i+1}\right) .
$$

Hence, $H\left(D, U^{*} ; \lambda_{k}\right)<0$ is equivalent to $k \in\{1,2, \cdots, i\}$.

Suppose on the contrary that (1.2) has no non-constant positive solution. Then by Lemma 4.1, we have

$$
\operatorname{index}\left(F(D ; \cdot), U^{*}\right)=-1
$$

since $\sigma=\sum_{k=1}^{i} m\left(\lambda_{k}\right)$ is odd. From (4.3)-(4.5) we get a contradiction. Therefore, $F(D ; U)=0$ has at least a positive solution that is different from the constant solution, that is, (1.2) has at least a non-constant positive solution and the proof is completed.

\section{Discussion and conclusion}

This paper presents the existence of the non-constant positive steady states of a generalized predator-prey system with cross-diffusion, where the cross-diffusion is included in such a way that the prey runs away from the predator. Based on the mathematical analysis, we find that the system has a non-constant positive steady state if either of the self-diffusions is large or the cross-diffusion is small. The theoretical analysis shows that 
the cross-diffusion phenomenon has the potential to play an important role in the coexistence information, even when the cross-diffusion coefficients are relatively small. The proposed approach has applicability to other reaction-diffusion systems including crossdiffusion, such as chemotaxis, food chain, and cell motility models. It is of great interest to us the development of a general mathematical framework that allows for the treatment of some generalized predator-prey systems, such as Gause model. From the biological point of view, the analysis gives a theoretical support for reaction-diffusion systems with cross-diffusion as viable mathematical models for studying coexistence phenomena.

There has been a growing understanding regarding the dynamics of real ecosystems. It is important to reveal the different dynamics regimes arising as a result of perturbation of the system parameters. For cross-diffusion models, further work is needed to state the dependence of cross-diffusion coefficients on the concentrations of the species. As biologists and mathematicians become more aware of the capacity of cross-diffusion to effect the population dynamics, and as they develop better intuition about how such processes occur, they are likely to be able to exploit cross-diffusion as a tool to design systems with desired properties rather than regarding it merely as an exotic phenomenon.

\section{Acknowledgments}

The authors are grateful to the referees for their valuable comments and advice on this manuscript.

\section{References}

[1] Y. Kuang, Global stability of Gause-type predator-prey systems, J. Math. Biol. 28 (1990) 463-474.

[2] K. Hasík, Uniqueness of limit cycle in the predator-prey system with symmetric prey isocline, Math. Biol. 164 (2000) 203-215.

[3] Y. Liu, Geometric criteria for the nonexistence of cycles in Gause-type predator-prey systems, Proc. Amer. Math. Soc. 133 (2005) 3619-3626.

[4] X. Ding, J. Jiang, Multiple periodic solutions in generalized Gause-type predator-prey systems with non-monotonic numerical responses, Nonlinear Anal. RWA 10 (2009) 2819-2827.

[5] K. Hasík, On a predator-prey system of Gause-type, J. Math. Biol. 60 (2010) 59-74.

[6] X. Chen, Y. Qi, M. Wang, A strongly coupled predator-prey system with nonmonotonic functional response, Nonlinear Anal. 67 (2007) 1966-1979.

[7] A. Ardito, P. Ricciardi, Lyapunov functions for a generalized Gause-type model, J. Math. Biol. 33 (1995) 816-828. 
[8] N.S. Goel, S.C. Maitra, On the Volterra and other nonlinear models of interacting populations, Rev. Mod. Phys. 43 (1971) 231-276.

[9] C.S. Holling, The functional response of predators to prey density and its role in mimicry and population regulation, Mem. Entomol. Soc. Can. 97 (1965) 5-60.

[10] J.H.P. Dawes, M.O. Souza, A derivation of Holling's type I, II and III functional responses in predator-prey systems, J. Theor. Biol. 327 (2013) 11-22.

[11] R.K. Ghaziani, W. Govaerts, C. Sonck, Resonance and bifurcation in a discretetime predator-prey system with Holling functional response, Nonlinear Anal. RWA 13 (2012) 1451-1465.

[12] H. Baek, A food chain system with Holling type IV functional response and impulsive perturbations, Comput. Math. Appl. 60 (2010) 1152-1163.

[13] R.E. Kooij, A. Zegeling, A Predator-Prey Model with Ivlev's Functional Response, J. Math. Anal. Appl. 198 (1996) 473-489.

[14] Y. Jia, A sufficient and necessary condition for the existence of positive solutions for a prey-predator system with Ivlev-type functional response, Appl. Math. Lett. 24 (2011) 1084-1088.

[15] J. Sugie, Two-parameter bifurcation in a predator-prey system of Ivlev type, J. Math. Anal. Appl. 217 (1998) 349-371.

[16] X. Zeng, Non-constant positive steady states of a prey-predator system with crossdiffusions, J. Math. Anal. Appl. 332 (2007) 989-1009.

[17] W. Ko, K. Ryu, Coexistence states of a nonlinear Lotka-Volterra type predator-prey model with cross-diffusion, Nonlinear Anal. 71 (2009) 1109-1115.

[18] J. Shi, Z. Xie, K. Little, Cross-diffusion induced instability and stability in reactiondiffusion systems, J. Appl. Anal. Comput. 1 (2011) 95-119.

[19] G. Gambino, M.C. Lombardo, M. Sammartino, Pattern formation driven by crossdiffusion in a 2D domain, Nonlinear Anal. RWA 14 (2013) 1755-1779.

[20] Y. Jia, J. Wu, H.-K. Xu, Positive solutions of a Lotka-Volterra competition model with cross-diffusion, Comput. Math. Appl. 68 (2014) 1220-1228.

[21] Y. Lou, W.-M. Ni, Diffusion, self-diffusion and cross-diffusion, J. Differential Equations 131 (1996) 79-131.

[22] W.-M. Ni, Diffusion, cross-diffusion and their spike-layer steady states, Notices Amer. Math. Soc. 45 (1998) 9-18.

[23] G.S. Sebestyén, I. Faragó, R. Horváth, R. Kersner, M. Klincsik, Stability of patterns and of constant steady states for a cross-diffusion system, J. Comput. Appl. Math. 293 (2015) 293-296. 
[24] M. Delgado, M. Montenegro, A. Suárez, A Lotka-Volterra symbiotic model with crossdiffusion, J. Differential Equations 246 (2009) 2131-2149.

[25] Y.-X Wang, W.-T. Li, Effect of cross-diffusion on the stationary problem of a diffusive competition model with a protection zone, Nonlinear Anal. RWA 14 (2013) 224-245.

[26] A. Madzvamuse, H.S. Ndakwo, R. Barreira, Cross-diffusion-driven instability for reaction-diffusion systems: analysis and simulations, J. Math. Biol. 27 (2014) 262292.

[27] N. Shigesada, K. Kawasaki, E. Teramoto, Spatial segregation of interacting species, J. Theoret. Biol. 79 (1979) 83-99.

[28] A. Okubo, Diffusion and Ecological Problems: Mathematical Models, SpringerVerlag, Berlin, 1980.

[29] C.-S. Lin, W.-M. Ni, I. Takagi, Large amplitude stationary solutions to a chemotaxis system, J. Differential Equations 72 (1988) 1-27.

[30] D. Gilbarg, N.S. Trudinger, Elliptic Partial Differential Equation of Second Order, Springer-Verlag, Berlin, 2001. 